\title{
A quasi-linear elliptic equation with critical growth on compact Riemannian manifold without boundary
}

\author{
João Marcos do Óa, Yunyan Yang ${ }^{\mathrm{b}}$ \\ ${ }^{a}$ Departamento de Matemática, Universidade Federal da Paraíba, 58051-900 João Pessoa, PB, Brazil \\ ${ }^{b}$ Department of Mathematics, Information School, Renmin University of China, Beijing 100872, P. R. China
}

\section{Abstract}

Let $(M, g)$ be an $N$-dimensional compact Riemannian manifold without boundary. When $m$ is a positive integer strictly smaller than $N$, we prove that

$$
\sup _{\|u\|_{m, N / m} \leq 1} \int_{M} e^{\alpha_{N, m}|u|^{N /(N-m)}} d v_{g}<\infty
$$

where $\|u\|_{m, N / m}$ is the usual Sobolev norm of $u \in W^{m, N / m}(M)$, and $\alpha_{N, m}$ is the best constant in Adams' original inequality (Ann. Math., 1988). This is a modified version of Adams' inequality on compact Riemannian manifold which has been proved by L. Fontana (Comment. Math Helv., 1993). Using the above inequality in the case when $m=1$, we establish sufficient conditions under which the quasilinear equation

$$
-\Delta_{N} u+\tau|u|^{N-2} u=f(x, u)
$$

has a nontrivial positive weak solution in $W^{1, N}(M)$, where $-\Delta_{N} u=-\operatorname{div}\left(|\nabla u|^{N-2} \nabla u\right), \tau>0$, and $f(x, u)$ behaves like $e^{\gamma|u|^{N /(N-1)}}$ as $|u| \rightarrow \infty$ for some $\gamma>0$.

Key words: Trudinger-Moser inequality, Adams inequality, Critical growth, $N$-Laplacian 2000 MSC: 58J05, 58E30, 35J60, 35B33

\section{Introduction and main results}

Let $(M, g)$ be a compact Riemannian manifold of dimension $N(N \geq 2)$ without boundary. Assume $m$ is a positive integer strictly smaller than $N$. Take $W^{m, N / m}(M)$ the usual Sobolev space, the completion of $C^{\infty}(M)$ under the norm

$$
\|u\|_{m, N / m}=\left(\int_{M}\left(\left|\nabla^{m} u\right|^{N / m}+|u|^{N / m}\right) d v_{g}\right)^{m / N}
$$

where $\nabla^{m} u=\Delta_{g}^{m / 2} u$ if $m$ is even, $\nabla \Delta_{g}^{(m-1) / 2} u$ if $m$ is odd, $\nabla, \Delta_{g}$ are the gradient operator and the Laplace-Beltrami operator respectively, $d v_{g}$ is the volume element of $(M, g)$. Precisely in local

URL: jmbo@mat.ufpb.br (João Marcos do Ó), yunyanyang@ruc.edu.cn (Yunyan Yang) Preprint submitted to $* * *$ 
coordinates $\left\{x^{i}\right\}_{i=1}^{N}, g=g_{i j}(x) d x^{i} d x^{j}, d v_{g}=\sqrt{g} d x^{1} \cdots d x^{N}$,

$$
\nabla f=g^{i j} \frac{\partial f}{\partial x^{j}} \frac{\partial}{\partial x^{i}}, \quad \Delta_{g} f=-\frac{1}{\sqrt{g}} \frac{\partial}{\partial x^{i}}\left(g^{i j} \sqrt{g} \frac{\partial f}{\partial x^{i}}\right)
$$

for all $f \in C^{\infty}(M)$, where $\left(g^{i j}\right)=\left(g_{i j}\right)^{-1}$, the inverse of the matrix $\left(g_{i j}\right)$, and $\sqrt{g}=\sqrt{\operatorname{det}\left(g_{i j}\right)}$. Here we have used the repeated summation convention.

In a celebrated paper [10], L. Fontana obtained the following estimates:

$$
\sup _{\int_{M} u d v_{g}=0,\left\|\nabla^{m} u\right\|_{N / m} \leq 1} \int_{M} e^{\alpha_{N, m}|u|^{N /(N-m)}} d v_{g}<\infty
$$

where $\|\cdot\|_{N / m}$ denotes the $L^{N / m}(M)$ norm and

$$
\alpha_{N, m}= \begin{cases}\frac{N}{\omega_{N-1}}\left(\frac{\pi^{N / 2} 2^{m} \Gamma\left(\frac{m+1}{2}\right)}{\Gamma\left(\frac{N-m+1}{2}\right)}\right)^{\frac{N}{N-m}} & \text { if } m \text { is odd } \\ \frac{N}{\omega_{N-1}}\left(\frac{\pi^{N / 2} 2^{m} \Gamma\left(\frac{m}{2}\right)}{\Gamma\left(\frac{N-m}{2}\right)}\right)^{\frac{N}{N-m}} & \text { if } m \text { is even. }\end{cases}
$$

If $\alpha_{N, m}$ is replaced by any larger number, the integral in (1.2) is still finite, but cannot be bounded uniformly by any constant. Inequality (1.2) is a manifold case of the well-known Adams inequality [1], which is the generalization of the Trudinger-Moser inequality [13, 15, 16]. Adams' approach to the problem is to express $u$ as the Riesz Potential of its gradient of order $m$ and then use the symmetrization to reduce the problem to one dimensional case. By estimating the asymptotic express of the Green function of $\Delta_{g}^{m}$, Fontana was able to find the counterpart of Adams' approach on $(M, g)$.

Replacing the hypothesis $\int_{M} u d v_{g}=0,\left\|\nabla^{m} u\right\|_{N / m} \leq 1$ by $\|u\|_{m, N / m} \leq 1$, we will show (1.2) is still valid. More generally, if (1.1) is replaced by an equivalent Sobolev norm

$$
\|u\|_{S_{m, \tau}}:=\left(\int_{M}\left(\left|\nabla^{m} u\right|^{N / m}+\tau|u|^{N / m}\right) d v_{g}\right)^{m / N}
$$

for any $\tau>0$, we have the following:

Theorem 1.1 Let $(M, g)$ be a compact Riemannian manifold of dimension $N$ without boundary and $m$ a positive strictly smaller than $N$. Then for any $\tau>0$

$$
\sup _{u \in W^{m, \frac{N}{m}}(M),\|u\|_{S, m} \leq 1} \int_{M} e^{\alpha_{N, m}|u|^{N /(N-m)}} d v_{g}<\infty,
$$

where $\alpha_{N, m}$ is defined by (1.3). Furthermore this inequality is sharp: when $\alpha_{N, m}$ is replaced by any larger number, the integral in (1.5) is still finite, but the supremum is infinity.

Theorem 1.1 is a modification of Fontana's result. But nevertheless, the inequality (1.5) will be more natural when we consider related partial differential equations. We remark that Theorem 1.1 is a generalization of our recent result [18]. The proof of Theorem 1.1 is based on (1.2) and the Young inequality in a nontrivial way. Similar idea has been used by Adimurthi and the second named author [3]. A special case of Theorem 1.1 is $m=1$, which is also known by Li 
[11], namely

Theorem 1.2 Let $(M, g)$ be a compact Riemannian manifold of dimension $N$ without boundary. Then for any $\tau>0$

$$
\sup _{u \in W^{1, N}(M),\|u\|_{1, \tau} \leq 1} \int_{M} e^{\alpha_{N}|u|^{N /(N-1)}} d v_{g}<\infty,
$$

where $\|u\|_{S_{1, \tau}}$ is defined by (1.4), $\alpha_{N}=\alpha_{N, 1}=N \omega_{N-1}^{1 /(N-1)}, \omega_{N-1}$ is the volume of the unit sphere $\mathbb{S}^{N-1}$. Furthermore this inequality is sharp: when $\alpha_{N}$ is replaced by any larger number, the integral in (1.6) is still finite, but the supremum is infinity.

Next we study the existence of solutions to the following quasi-linear equation:

$$
\left\{\begin{array}{l}
-\Delta_{N} u+\tau|u|^{N-2} u=f(x, u) \text { in } \quad M \\
u \geq 0 \quad \text { in } \quad M
\end{array}\right.
$$

where $-\Delta_{N} u=-\operatorname{div}_{\mathrm{g}}\left(|\nabla u|^{N} \nabla u\right)$, the nonlinearity $f(x, u)$ has the maximal growth on $u$ which allows us to treat problem (1.7) variationally in the Sobolev space $W^{1, N}(M)$. Motivated by pioneer works of Adimurthi [2], de Figueiredo et al. [7, 8], do Ó [9], we say that a function $f: M \times \mathbb{R} \rightarrow \mathbb{R}$ has subcritical growth on $M$ if for any $\alpha>0$

$$
\lim _{|s| \rightarrow \infty} \frac{f(x, s)}{e^{\alpha|s|^{\mid /(N-1)}}}=0 \quad \text { uniformly for } \quad x \in M
$$

and $f$ has critical growth on $M$ if there exists $\alpha_{0}>0$ such that

$$
\lim _{|s| \rightarrow \infty} \frac{|f(x, s)|}{e^{\alpha|s|} \mid(N / N)}=\left\{\begin{array}{l}
0 \text { uniformly for } x \in M, \forall \alpha>\alpha_{0} \\
\infty, \quad \forall \alpha<\alpha_{0} .
\end{array}\right.
$$

In order to study the existence of solutions to equation (1.7), we assume $f$ satisfies the following:

$\left(H_{1}\right) f: M \times \mathbb{R} \rightarrow \mathbb{R}$ is continuous.

$\left(H_{2}\right)$ There exist $R>0$ and $A>0$ such that for all $s \geq R$ and all $x \in M$,

$$
0<F(x, s)=\int_{0}^{s} f(x, t) d t \leq A f(x, s) .
$$

$\left(H_{3}\right) f(x, s) \geq 0$ for all $(x, s) \in M \times[0, \infty)$ and $f(x, 0)=0$ for all $x \in M$.

The existence results of equation (1.7) in the subcritical case and critical case can be stated respectively as below.

Theorem 1.3 (The subcritical case) Assume $\left(H_{1}\right),\left(H_{2}\right),\left(H_{3}\right)$, and that $f$ has subcritical growth. Furthermore suppose that

$\left(H_{4}\right) \quad \lim \sup _{s \rightarrow 0^{+}} \frac{N F(x, s)}{s^{N}}<\tau$ uniformly for $x \in M$.

Then equation (1.7) has a nontrivial solution. 
Theorem 1.4 (The critical case) Assume $\left(H_{1}\right),\left(H_{2}\right),\left(H_{3}\right)$ and that $f$ has critical growth. Furthermore suppose $\left(\mathrm{H}_{4}\right)$ and

$\left(H_{5}\right)$

$$
s f(x, s) e^{-\alpha_{0} s^{N /(N-1)}} \rightarrow+\infty \text { as } s \rightarrow+\infty \text { uniformly for } x \in M .
$$

Then equation (1.7) has a nontrivial solution.

Let us explain the relation between Theorem 1.2 and Theorems 1.3 and 1.4. Solutions to equation (1.7) are critical points of the functional

$$
J(u):=\frac{1}{N} \int_{M}\left(|\nabla u|^{N}+\tau|u|^{N}\right) d v_{g}-\int_{M} F(x, u) d v_{g},
$$

where $F(x, s)=\int_{0}^{s} f(x, t) d t$ for all $x \in M$ and $s \in \mathbb{R}$. In view of the structure of $J$, particularly its first term $\int_{M}\left(|\nabla u|^{N}+\tau|u|^{N}\right) d v_{g}$, it is reasonable to use Theorem 1.2 instead of Fontana's original inequality (1.2) to study the compactness of the Palais-Smale sequence of $J$. This is exactly our motivation of establishing Theorem 1.2, more generally Theorem 1.1.

The proofs of Theorems 1.3 and 1.4 are based on the Mountain Pass theory. Similar idea has been used by de Figueiredo et al. [8] to establish the same results in the case when $(M, g)$ is replaced by any smooth bounded domain in $\mathbb{R}^{2}$.

The remaining part of the paper is organized as following: In Section 2, we prove Theorem 1.1, particularly Theorem 1.2. As an application of Theorem 1.2, Theorems 1.3 and 1.4 will be proved in Section 3. In Section 4, we will give an example of critical points not satisfying $\left(H_{4}\right)$.

\section{Proof of Theorem 1.1}

In this section we will prove Theorem 1.1. The method we used here is combining Fontana's inequality (1.2) and the Young inequality. The proof is straightforward and divided into two steps:

Step 1: For any $0<m<N, \alpha_{N, m}$ is the largest possible constant such that the integrals in (1.5) are uniformly bounded.

Based on Fontana's result, the integral in (1.5) in our case is still finite if $\alpha_{N, m}$ is replaced by any larger number. However we are left to prove $\alpha_{N, m}$ is the largest possible constant such that the integrals in (1.5) are uniformly bounded under the hypothesis $\|u\|_{S_{m, \tau}} \leq 1$. Following Adams [1] and Fontana [10], we distinguish two cases:

Case 1: $m=1$. In this case, $\alpha_{N, 1}=N \omega_{N-1}^{\frac{1}{N-1}}$. For some point $p \in M$, let $r=r(x)=d_{g}(p, x)$ be the geodesic distance between $x$ and $p$. Without loss of generality we assume the injective radius of $(M, g)$ is strictly larger than 1 . Set

$$
\phi_{\delta}(x)=\left\{\begin{array}{lll}
1, & \text { when } & r<\delta \\
\left(\log \frac{1}{\delta}\right)^{-1} \log \frac{1}{r}, & \text { when } & \delta \leq r \leq 1 \\
0, & \text { when } & r>1
\end{array}\right.
$$


Then $\phi_{\delta} \in W^{1, N}(M)$ and for any $\tau>0$

$$
\int_{M}\left(\left|\nabla \phi_{\delta}\right|^{N}+\tau\left|\phi_{\delta}\right|^{N}\right) d v_{g}=\left(\log \frac{1}{\delta}\right)^{1-N} \omega_{N-1}\left(1+O\left(\frac{1}{\log \delta}\right)\right) .
$$

Denote $\widetilde{\phi}_{\delta}=\phi_{\delta} /\left\|\phi_{\delta}\right\|_{S_{1, \tau}}$. Then we have on the geodesic ball $B_{p}(\delta) \subset M$,

$$
\left.\widetilde{\phi}_{\delta}\right|^{\frac{N}{N-1}}=\left(\log \frac{1}{\delta}\right) \omega_{N-1}^{-\frac{1}{N-1}}\left(1+O\left(\frac{1}{\log \delta}\right)\right) .
$$

It follows immediately that for any $\gamma>N \omega_{N-1}^{1 /(N-1)}$, as $\delta \rightarrow 0$,

$$
\int_{M} e^{\gamma\left|\widetilde{\phi}_{\delta}\right| N-N} d v_{g} \geq \int_{B_{p}(\delta)} e^{\gamma\left|\widetilde{\phi}_{\delta}\right| N-1} d v_{g} \rightarrow+\infty .
$$

Case 2: $m>1$. Let $\Phi \in C^{\infty}[0,1]$ be such that

$$
\Phi(0)=\Phi^{\prime}(0)=\cdots=\Phi^{(m-1)}(0)=0, \quad \Phi(1)=\Phi^{\prime}(1)=1
$$

and if $m>2$,

$$
\Phi^{\prime \prime}(1)=\cdots=\Phi^{(m-1)}(1)=0 .
$$

For any fixed small $\epsilon>0$, we set

$$
H(t)=\left\{\begin{array}{lll}
\epsilon \Phi\left(\frac{t}{\epsilon}\right) & \text { when } & 0 \leq t \leq \epsilon \\
t & \text { when } & \epsilon<t \leq 1-\epsilon \\
1-\epsilon \Phi\left(\frac{1-t}{\epsilon}\right) & \text { when } & 1-\epsilon<t \leq 1 \\
1 & \text { when } & t>1 .
\end{array}\right.
$$

For $0<\delta<1,0<t<1$, we define

$$
\Psi(t)=H\left(\left(\log \frac{1}{\delta}\right)^{-1} \log \frac{1}{t}\right) .
$$

For any fixed point $p \in M$, denote the distance between $p$ and $x$ by $r=r(x)=d_{g}(p, x)$, then the function

$$
\phi_{\delta}(x)=\Psi(r) \in C^{m}\left(B_{p}(1)\right) .
$$

By a delicate calculation of Fontana ([10], pages 441-443),

$$
\int_{M}\left|\nabla^{m} \phi_{\delta}\right|^{\frac{n}{m}} d v_{g} \leq c(m, N)^{\frac{N}{m}} \omega_{N-1}\left(1+C \epsilon+O\left(\frac{1}{\log \delta}\right)\right)\left(\log \frac{1}{\delta}\right)^{-(N-m) / m},
$$

where

$$
c(m, N)=\left\{\begin{array}{lll}
2^{\frac{m-2}{2}} \Gamma\left(\frac{m}{2}\right)(N-m)(N-m+2) \cdots(N-2) & \text { for } m \text { even } \\
2^{\frac{m-1}{2}} \Gamma\left(\frac{m+1}{2}\right)(N-m+1)(N-m+3) \cdots(N-2) & \text { for } m \text { odd. } \\
5 &
\end{array}\right.
$$


We are left to estimate

$$
\int_{M}\left|\phi_{\delta}\right|^{\frac{N}{m}} d v_{g}=\int_{0}^{1}\left(H\left(\frac{\log s}{\log \delta}\right)\right)^{\frac{N}{m}} \omega_{N-1} s^{N-1}(1+O(s)) d s .
$$

Since $H(t) \leq C t$, we obtain

$$
\int_{M}\left|\phi_{\delta}\right|^{\frac{N}{m}} d v_{g}=O\left(\left(\log \frac{1}{\delta}\right)^{-\frac{N}{m}}\right) .
$$

Define $\widetilde{\phi}_{\delta}=\phi_{\delta} /\left\|\phi_{\delta}\right\|_{S_{m, \tau}}$. Then we have on the geodesic ball $B_{p}(\delta)$,

$$
\left|\widetilde{\phi}_{\delta}\right|^{\frac{N}{N-m}} \geq\left(\log \frac{1}{\delta}\right) \frac{1-C \epsilon+O\left(\frac{1}{\log \delta}\right)}{\omega_{N-1}^{\frac{m}{N-m}} c(m, N)^{\frac{N}{N-m}}}\left(1+O\left(\frac{1}{\log \delta}\right)\right) .
$$

It is easy to see that for any $\gamma>N \omega_{N-1}^{\frac{m}{N-m}} c(m, N)^{\frac{N}{N-m}}=\alpha_{N, m}$,

$$
\int_{M} e^{\left.\gamma \widetilde{\phi}_{\delta}\right|^{N-m}} d v_{g} \geq \int_{B_{p}(\delta)} e^{\gamma\left|\widetilde{\phi}_{\delta}\right|^{\frac{N}{N-m}}} d v_{g} \rightarrow+\infty
$$

as $\delta \rightarrow 0$, provided that $\epsilon$ is chosen sufficiently small. This completes the proof of Step 1 .

Step 2: The modified Adams inequality (1.5) holds.

In view of Fontana's inequality, to conclude (1.5), one only needs to prove

$$
\sup _{u \in W^{m, \frac{N}{m}}(M),\|u\|_{S_{m, \tau}} \leq 1} \int_{|u-\bar{u}| \geq \bar{u}>0} e^{\alpha_{N, m}|u|^{N /(N-m)}} d v_{g}<\infty .
$$

Assume $\|u\|_{S_{m, \tau}} \leq 1$. Denote $\bar{u}=\frac{1}{\operatorname{Vol}(M)} \int_{M} u d v_{g}$ and write $u=(u-\bar{u})+\bar{u}$. Clearly $\bar{u}$ is bounded. Using an elementary inequality $(a+b)^{p} \leq b^{p}+\left(2^{p}-1\right) b^{p-1} a$ for $0 \leq a \leq b$ and $p>1$, one has by employing the Young inequality

$$
(a+b)^{p} \leq(1+\gamma) b^{p}+c(p) \frac{a^{p}}{\gamma^{p-1}}, \quad \forall \gamma>0,
$$

where $c(p)$ is a constant depending only on $p$. Taking $a=\bar{u}, b=|u-\bar{u}|, p=N /(N-m), \gamma$ satisfies

$$
1+\gamma=\left(\int_{M}\left|\nabla^{m} u\right|^{\frac{N}{m}} d v_{g}\right)^{1-p}
$$

and $w=(1+\gamma)^{1 / p}(u-\bar{u})$. Then one can see

$$
\int_{M}\left|\nabla^{m} w\right|^{\frac{N}{m}} d v_{g}=1, \quad \int_{M} w d v_{g}=0 .
$$

Since

$$
\gamma=\left(1-\tau \int_{M}|u|^{\frac{N}{m}} d v_{g}\right)^{1-p}-1 \geq \tau(p-1) \int_{M}|u|^{\frac{N}{m}} d v_{g}
$$

and on the set $\{x \in M:|u(x)-\bar{u}| \geq \bar{u}>0\}$,

$$
|u|^{p} \leq|w|^{p}+c(p) \frac{\bar{u}^{p}}{\gamma^{p-1}},
$$

one ends step 2 by using Fontana's inequality and completes the proof of Theorem 1.1. 


\section{Applications of Theorem 1.1}

In this section we will use the Mountain Pass theory to establish Theorems 1.3 and 1.4. To this end, we begin with constructing a functional closely related to equation (1.7).

For $m \in \mathbb{N}, 0<m<N$, we assume $f: M \times \mathbb{R} \rightarrow \mathbb{R}$ is a continuous function and there exist constants $\beta>0, C>0$ such that

$$
|f(x, s)| \leq C e^{\beta|s| \frac{N}{N-m}}, \quad \forall(x, s) \in M \times \mathbb{R} .
$$

Let $F(x, s)=\int_{0}^{s} f(x, t) d t$. For $0<m<N$ and $\tau>0$, we define functionals

$$
J_{m, \tau}(u)=\frac{m}{N} \int_{M}\left(\left|\nabla^{m} u\right|^{N / m}+\tau|u|^{N / m}\right) d v_{g}-\int_{M} F(x, u) d v_{g}, \quad \forall u \in W^{m, \frac{N}{m}}(M) .
$$

In view of Theorem 1.1, $J_{m, \tau}$ is well defined on $W^{m, \frac{N}{m}}(M)$. When $m=1, J_{1, \tau}$ is exactly $J$ defined by (1.10). Clearly $J \in C^{1}\left(W^{1, N}(M), \mathbb{R}\right)$ and (3.1) becomes

$$
|f(x, s)| \leq C e^{\beta|s| \frac{N}{N-1}}, \quad \forall(x, s) \in M \times \mathbb{R} .
$$

\subsection{The geometry of the functional $\mathrm{J}$}

Define two functions

$$
\widetilde{f}(x, s)=\left\{\begin{array}{lll}
f(x, s) & \text { when } & (x, s) \in M \times(0, \infty) \\
0 & \text { when } & (x, s) \in M \times(-\infty, 0]
\end{array}\right.
$$

and $\widetilde{F}(x, s)=\int_{0}^{s} \widetilde{f}(x, t) d t$. If $f$ satisfies $\left(H_{1}\right)-\left(H_{5}\right)$, then so does $\widetilde{f}$. Moreover if $u \in W^{1, N}(M)$ is a solution of

$$
\left\{\begin{array}{l}
-\Delta_{N} u+\tau|u|^{N-2} u=\widetilde{f}(x, u) \text { in } M \\
u \geq 0 \text { in } M,
\end{array}\right.
$$

then it is also a solution of (1.7). Without loss of generality, we can assume henceforth that $f(x, s) \equiv 0$ for all $(x, s) \in M \times(-\infty, 0]$.

Lemma 3.1 Assume $\left(H_{1}\right),\left(H_{2}\right),\left(H_{3}\right)$, and (3.2). Then $J(t u) \rightarrow-\infty$ as $t \rightarrow+\infty$, for all $u \in W^{1, N}(M) \backslash\{0\}$ with $u \geq 0$.

Proof. Assume $u \in W^{1, N}(M) \backslash\{0\}$ with $u \geq 0$. By $\left(H_{2}\right)$, for $p>N$, there exist two positive constants $c_{1}$ and $c_{2}$ such that

$$
F(x, u) \geq c_{1} u^{p}-c_{2} .
$$

Hence

$$
J(t u) \leq \frac{t^{N}}{N} \int_{M}\left(|\nabla u|^{N}+|u|^{N}\right) d v_{g}-c_{1} t^{p} \int_{M}|u|^{p} d v_{g}+c_{2} .
$$

Since $p>N, J(t u) \rightarrow-\infty$ as $t \rightarrow+\infty$. 
Lemma 3.2 Assume $\left(H_{1}\right),\left(H_{4}\right)$, and (3.2). Then there exist $\delta, \sigma>0$ such that

$$
J(u) \geq \delta \quad \text { if } \quad\|u\|_{S_{1, \tau}}=\sigma .
$$

Proof. By $\left(H_{1}\right),\left(H_{4}\right)$, and (3.2), there exists some $\lambda<\tau$ such that for $q>N$

$$
F(x, u) \leq \frac{1}{N} \lambda|u|^{N}+C|u|^{q} e^{\beta|u|^{N-1}} \text { for all }(x, u) \in M \times \mathbb{R} .
$$

By Theorem 1.1 and the Hölder inequality,

$$
\begin{aligned}
\int_{M}|u|^{q} e^{\beta|u|^{\frac{N}{N-1}}} d v_{g} & \leq\left(\int_{M} e^{p^{\prime} \beta|u|^{N-1}} d v_{g}\right)^{\frac{1}{p^{\prime}}}\left(\int_{M}|u|^{q p} d v_{g}\right)^{\frac{1}{p}} \\
& \leq C\left(\int_{M}|u|^{q p} d v_{g}\right)^{\frac{1}{p}}
\end{aligned}
$$

provided that $\|u\|_{S_{1, \tau}} \leq \varrho$, where $p^{\prime} \beta \varrho^{\frac{N}{N-1}} \leq \alpha_{N}$ and $\frac{1}{p^{\prime}}+\frac{1}{p}=1$. Obviously

$$
\int_{M}|u|^{N} d v_{g} \leq \frac{1}{\tau}\|u\|_{S_{1, \tau}}^{N}, \quad \forall u \in W^{1, N}(M) \backslash\{0\} .
$$

This together with (3.3) and (3.4) implies that

$$
J(u) \geq \frac{1}{N}\left(1-\frac{\lambda}{\tau}\right)\|u\|_{S_{1, \tau}}^{N}-C\|u\|_{S_{1, \tau}}^{q} .
$$

Thus we can further choose $\sigma<\varrho$ and $\delta>0$ such that $J(u) \geq \delta$ if $\|u\|_{S_{1, \tau}}=\sigma$.

\subsection{Minimax level}

To get a more precise information of the minimax level obtained by the mountain pass theorem, we employ the Moser function sequence

$$
\mathbf{M}_{n}(x, r)=\frac{1}{\omega_{N-1}^{1 / N}}\left\{\begin{array}{lll}
(\log n)^{(N-1) / N} & \text { when } \quad r \leq R / n, \\
(\log n)^{-1 / N} \log (R / r) & \text { when } \quad R / n \leq r \leq R, \\
0 & \text { when } \quad r \geq R,
\end{array}\right.
$$

where $0<R<\operatorname{inj}(M), \operatorname{inj}(M)$ is the injective radius of $(M, g)$, and $r=r(x)$ denotes the geodesic distance between $x$ and a fixed point $O \in M$.

Lemma 3.3 Assume $\left(H_{1}\right),\left(H_{2}\right),\left(H_{3}\right)$, and $\left(H_{5}\right)$ hold. Then there exists $n \in \mathbb{N}$ such that

$$
\max _{t \geq 0} J\left(t \mathbf{M}_{n}\right)<\frac{1}{N}\left(\frac{\alpha_{N}}{\alpha_{0}}\right)^{N-1} .
$$

Proof. Suppose not. Then we have for all $n$

$$
\max _{t \geq 0} J\left(t \mathbf{M}_{n}\right) \geq \frac{1}{N}\left(\frac{\alpha_{N}}{\alpha_{0}}\right)^{N-1} .
$$


By Lemma 3.1, there exists $t_{n}>0$ for any fixed $n$ such that

$$
J\left(t_{n} \mathbf{M}_{n}\right)=\frac{1}{N} t_{n}^{N}\left\|\mathbf{M}_{n}\right\|_{S_{1, \tau}}^{N}-\int_{M} F\left(x, t_{n} \mathbf{M}_{n}\right) d v_{g}=\max _{t \geq 0} J\left(t \mathbf{M}_{n}\right) .
$$

Since $F(x, s) \geq 0$ for all $(x, s) \in M \times \mathbb{R}$, we get by combining (3.5) and (3.6) that

$$
t_{n}^{N}\left\|\mathbf{M}_{n}\right\|_{S_{1, \tau}}^{N} \geq\left(\frac{\alpha_{N}}{\alpha_{0}}\right)^{N-1}
$$

By (3.6), we arrive at $\frac{d}{d t} J\left(t \mathbf{M}_{n}\right)=0$ at $t=t_{n}$, or equivalently

$$
t_{n}^{N}\left\|\mathbf{M}_{n}\right\|_{S_{1, \tau}}^{N}=\int_{M} t_{n} \mathbf{M}_{n} f\left(x, t_{n} \mathbf{M}_{n}\right) d v_{g}
$$

By $\left(H_{5}\right), \forall \rho>0, \exists R_{\rho}>0$ such that for all $s \geq R_{\rho}$, there holds

$$
s f(x, s) \geq \rho e^{\alpha_{0} s^{\frac{N}{N-1}}} .
$$

Choosing a normal coordinate system near the point $O$, we calculate

$$
\begin{aligned}
\int_{M}\left|\nabla \mathbf{M}_{n}\right|^{N} d v_{g} & =\frac{1}{\omega_{N-1} \log n} \int_{\frac{R}{n}}^{R} \frac{\omega_{N-1}}{r}\left(1+O\left(r^{2}\right)\right) d r \\
& =1+\frac{O\left(R^{2}\right)}{\log n}
\end{aligned}
$$

and similarly

$$
\int_{M} \tau\left|\mathbf{M}_{n}\right|^{N} d v_{g}=\frac{1}{\log n}\left(o_{n}(1)+O\left(R^{2}\right)\right),
$$

where $o_{n}(1) \rightarrow 0$ as $n \rightarrow \infty$ and $\left|O\left(R^{2}\right)\right| \leq C R^{2}$. Hence we get

$$
\left\|\mathbf{M}_{n}\right\|_{S_{1, \tau}}^{N}=1+\frac{1}{\log n}\left(o_{n}(1)+O\left(R^{2}\right)\right) \text {. }
$$

Thus (3.7) becomes

$$
t_{n}^{N} \geq\left(\frac{\alpha_{N}}{\alpha_{0}}\right)^{N-1}\left(1+\frac{o_{n}(1)+O\left(R^{2}\right)}{\log n}\right) .
$$

This together with (3.8) and (3.9) implies

$$
\begin{aligned}
t_{n}^{N}\left\|\mathbf{M}_{n}\right\|_{S_{1, \tau}}^{N} & \geq \rho \int_{B_{R / n}(O)} e^{\alpha_{0}\left|t_{n} \mathbf{M}_{n}\right|^{\frac{N}{N-1}}} d v_{g} \\
& =\rho \frac{\omega_{N-1}}{N}\left(\frac{R}{n}\right)^{N} e^{\alpha_{0} t_{n}^{\frac{N}{N-1}} \omega_{N-1}^{-\frac{1}{N-1}} \log n}\left(1+O\left(\frac{R^{2}}{n^{2}}\right)\right)
\end{aligned}
$$

for sufficiently large $n$. The power of this inequality is evident. Since $\left\|\mathbf{M}_{n}\right\|_{S_{1, \tau}}^{N}$ is bounded and $\rho>0$, it is easy to see from (3.12) that $t_{n}$ is a bounded sequence. Notice that $t_{n}^{N /(N-1)}>\alpha_{N} / \alpha_{0}$ implies $\alpha_{0} t_{n}^{N /(N-1)} \omega_{N-1}^{-1 /(N-1)}>N$, then it follows from (3.11) and (3.12) that

$$
\lim _{n \rightarrow \infty} t_{n}^{N}=\left(\frac{\alpha_{N}}{\alpha_{0}}\right)^{N-1}
$$


It follows from (3.11) and (3.12) that

$$
\begin{aligned}
t_{n}^{N}\left\|\mathbf{M}_{n}\right\|_{S_{1, \tau}}^{N} & \geq \rho \frac{\omega_{N-1}}{N}\left(\frac{R}{n}\right)^{N} e^{N \log n}\left(1+o_{n}(1)+O\left(R^{2}\right)\right) \\
& =\rho \frac{\omega_{N-1}}{N} R^{N}\left(1+o_{n}(1)+O\left(R^{2}\right)\right) .
\end{aligned}
$$

By (3.10) and (3.13), letting $n \rightarrow \infty$ in the above inequality, we obtain

$$
\left(\frac{\alpha_{N}}{\alpha_{0}}\right)^{N-1} \geq \rho \frac{\omega_{N-1}}{N} R^{N}\left(1+O\left(R^{2}\right)\right) .
$$

This is impossible when $\rho$ is chosen sufficiently large and completes the proof of the Lemma.

\subsection{Palais-Smale sequences}

We state a manifold version of Lemma 2.1 in [8] as below. Since the proof is almost the same, we omit the details.

Lemma 3.4 Let $u_{n} \rightarrow u$ in $L^{1}(M)$. Assume that $f\left(x, u_{n}(x)\right)$ and $f(x, u(x))$ are also $L^{1}(M)$ functions. If $\int_{M}\left|f\left(x, u_{n}(x)\right) u_{n}(x)\right| d v_{g} \leq C$, then $f\left(x, u_{n}\right) \rightarrow f(x, u)$ in $L^{1}(M)$.

The following result can be found in $[5,6]$.

Lemma 3.5 (Cherrier) Let $W$ be any compact $N$-dimensional Riemannian manifold with smooth boundary $\partial W$. Then for any $\alpha<\alpha_{N} / 2^{1 /(N-1)}$,

$$
\sup _{\|\nabla v\|_{L^{N}(W)} \leq 1, \int_{W} v d v_{g}=0} \int_{W} e^{\alpha|v|^{N-1}} d v_{g}<\infty .
$$

Moreover when $\alpha>\alpha_{N} / 2^{1 /(N-1)}$, the above integral is still finite, but the supremum is infinite.

We remark that the significance of Lemma 3.5 is that the best constant $\alpha_{N} / 2^{1 /(N-1)}$ depends only on the dimension of $W$. When $N=2$, this result has been strengthened by the second author in [17].

Lemma 3.6 Assume $f$ satisfies $\left(H_{1}\right),(3.2)$, and there exist $R_{0}>0, \mu>N$ such that

$$
0 \leq \mu F(x, s) \leq s f(x, s), \quad \forall|s| \geq R_{0}, \forall x \in M .
$$

Let $\left(u_{n}\right) \subset W^{1, N}(M)$ be a Palais-Smale sequence of any level, i.e., $J\left(u_{n}\right) \rightarrow c, J^{\prime}\left(u_{n}\right) \rightarrow 0$ in $W^{-1, \frac{N}{N-1}}(M)$ as $n \rightarrow \infty$. Then there exists a subsequence of $\left(u_{n}\right)$, still denoted by $\left(u_{n}\right)$, and $u \in W^{1, N}(M)$ such that

$$
\begin{cases}f\left(x, u_{n}\right) \rightarrow f(x, u) & \text { in } \quad L^{1}(M) \\ \nabla u_{n}(x) \rightarrow \nabla u(x) & \text { for almost all } x \in M \\ \left|\nabla u_{n}\right|^{N-2} \nabla u_{n} \rightarrow|\nabla u|^{N-2} \nabla u & \text { weakly in }\left(L^{N /(N-1)}(M)\right)^{N}\end{cases}
$$


Proof. Assume $\left(u_{n}\right) \subset W^{1, N}(M)$ be a Palais-Smale sequence of any level, i.e.,

$$
\begin{gathered}
\frac{1}{N} \int_{M}\left(\left|\nabla u_{n}\right|^{N}+\tau\left|u_{n}\right|^{N}\right) d v_{g}-\int_{M} F\left(x, u_{n}\right) d v_{g} \rightarrow c, \\
\left|\left\langle J^{\prime}\left(u_{n}\right), \varphi\right\rangle\right| \leq \tau_{n}\|\varphi\|_{S_{1, \tau}}, \quad \forall \varphi \in W^{1, N}(M),
\end{gathered}
$$

where $\tau_{n} \rightarrow 0$ as $n \rightarrow \infty$. Multiplying (3.15) by $\mu$ and subtracting (3.16) with $\varphi=u_{n}$, we obtain

$$
\left(\frac{\mu}{N}-1\right)\left\|u_{n}\right\|_{S_{1, \tau}}^{N}-\int_{M}\left(\mu F\left(x, u_{n}\right)-u_{n} f\left(x, u_{n}\right)\right) d v_{g} \leq C+\tau_{n}\left\|u_{n}\right\|_{S_{1, \tau}}
$$

for some constant $C$. By (3.14) and $\left(H_{1}\right)$, the second term in the above inequality has lower bound, and thus $u_{n}$ is bounded in $W^{1, N}(M)$. It then follows that

$$
\left.\left.\int_{M}|| \nabla u_{n}\right|^{N-2} \nabla u_{n}\right|^{\frac{N}{N-1}} d v_{g} \leq C, \int_{M} F\left(x, u_{n}\right) d v_{g} \leq C, \text { and } \int_{M} f\left(x, u_{n}\right) u_{n} d v_{g} \leq C .
$$

Moreover, up to a subsequence, we may assume

$$
\begin{aligned}
& u_{n} \rightarrow u \text { weakly in } W^{1, N}(M), u_{n} \rightarrow u \text { a. e. in } M \\
& u_{n} \rightarrow u \text { strongly in } L^{q}(M), \forall q \geq 1 .
\end{aligned}
$$

The assumption (3.14) implies that $s f(x, s)=|s f(x, s)|$ for all $s \geq R_{0}$, and thus

$$
\int_{M}\left|f\left(x, u_{n}\right) u_{n}\right| d v_{g} \leq C .
$$

It then follows from Lemma 3.4 that $f\left(x, u_{n}\right) \rightarrow f(x, u)$ in $L^{1}(M)$.

Next we will prove $\nabla u_{n}(x) \rightarrow \nabla u(x)$ almost everywhere. Up to a subsequence, we can define an energy concentration set for some $\delta>0$ to be determined later,

$$
\Sigma_{\delta}=\left\{x \in M: \lim _{r \rightarrow 0} \lim _{n \rightarrow \infty} \int_{B_{r}(x)}\left(\left|\nabla u_{n}\right|^{N}+\tau\left|u_{n}\right|^{N}\right) d v_{g} \geq \delta\right\} .
$$

Since $\left(u_{n}\right)$ is bounded in $W^{1, N}(M), \Sigma_{\delta}$ must be a finite set. For any $x^{*} \in M \backslash \Sigma_{\delta}$, there exists $r: 0<r<\operatorname{dist}\left(x^{*}, \Sigma_{\delta}\right)$ such that

$$
\lim _{n \rightarrow \infty} \int_{B_{r}\left(x^{*}\right)}\left(\left|\nabla u_{n}\right|^{N}+\tau\left|u_{n}\right|^{N}\right) d v_{g}<\delta .
$$

It follows that for large $n$,

$$
\int_{B_{r}\left(x^{*}\right)}\left(\left|\nabla u_{n}\right|^{N}+\tau\left|u_{n}\right|^{N}\right) d v_{g}<\delta
$$

Let $\overline{u_{n}}=\int_{B_{r}\left(x^{*}\right)} u_{n} d v_{g}$. It is easy to see from (3.17) that $\left|\overline{u_{n}}\right| \leq \delta^{1 / N}(\operatorname{Vol}(M))^{1-1 / N}$, and thus

$$
\begin{aligned}
\int_{B_{r}\left(x^{*}\right)} e^{\beta\left|u_{n}\right|^{\frac{N}{N-1}}} d v_{g} & \leq \int_{B_{r}\left(x^{*}\right)} e^{2 \frac{N}{N-1} \beta\left|u_{n}-\overline{u_{n}}\right|^{\frac{N}{N-1}}+2 \frac{N}{N-1} \beta\left|\bar{u}_{n}\right|^{\frac{N}{N-1}}} d v_{g} \\
& \leq C \int_{B_{r}\left(x^{*}\right)} e^{2 \frac{N}{N-1} \beta\left|u_{n}-\overline{u_{n}}\right| \frac{N}{N-1}} d v_{g} .
\end{aligned}
$$


Now we choose $\delta$ such that $2^{\frac{N}{N-1}} \beta \delta^{\frac{1}{N-1}}<\alpha_{N} / 2^{\frac{1}{N-1}}$. Then $e^{\beta\left|u_{n}\right| \frac{N}{N-1}}$ is bounded in $L^{q}\left(B_{r}\left(x^{*}\right)\right)$ for some $q>1$, thanks to Lemma 3.5. By (3.2), $f\left(x, u_{n}\right)$ is also bounded in $L^{q}\left(B_{r}\left(x^{*}\right)\right)$. For any $\eta>0$, denote

$$
A_{\eta}=\left\{x \in B_{r}\left(x^{*}\right):|u(x)| \geq \eta\right\} .
$$

We estimate

$$
\begin{aligned}
\int_{A_{\eta}}\left|f\left(x, u_{n}\right)-f(x, u) \| u\right| d v_{g} & \leq\left(\int_{A_{\eta}}\left|f\left(x, u_{n}\right)-f(x, u)\right|^{q} d v_{g}\right)^{1 / q}\left(\int_{A_{\eta}}|u|^{q^{\prime}}\right)^{1 / q^{\prime}} \\
& \leq C\left(\int_{A_{\eta}}|u|^{q^{\prime}}\right)^{1 / q^{\prime}}
\end{aligned}
$$

where $1 / q+1 / q^{\prime}=1$, since $f\left(x, u_{n}\right)$ is bounded in $L^{q}\left(B_{r}\left(x^{*}\right)\right)$. Hence for any $v>0$,

$$
\int_{A_{\eta}}\left|f\left(x, u_{n}\right)-f(x, u) \| u\right| d v_{g}<v,
$$

provided that $\eta$ is chosen sufficiently large. Since $f\left(x, u_{n}\right) \rightarrow f(x, u)$ in $L^{1}(M)$,

$$
\lim _{n \rightarrow \infty} \int_{B_{r}\left(x^{*}\right) \backslash A_{\eta}}\left|f\left(x, u_{n}\right)-f(x, u) \| u\right| d v_{g}=0 .
$$

Combining (3.18) and (3.19), we have

$$
\lim _{n \rightarrow \infty} \int_{B_{r}\left(x^{*}\right)}\left|f\left(x, u_{n}\right)-f(x, u) \| u\right| d v_{g} \leq v .
$$

Since $v>0$ is arbitrary, we obtain

$$
\lim _{n \rightarrow \infty} \int_{B_{r}\left(x^{*}\right)}\left|f\left(x, u_{n}\right)-f(x, u) \| u\right| d v_{g}=0 .
$$

On the other hand, we have by using the Hölder inequality,

$$
\int_{B_{r}\left(x^{*}\right)}\left|f\left(x, u_{n}\right)\left\|u_{n}-u \mid d v_{g} \leq\right\| f\left(x, u_{n}\right)\left\|_{L^{q}\left(B_{r}\left(x^{*}\right)\right)}\right\| u_{n}-u \|_{L^{q^{\prime}}(M)} \rightarrow 0,\right.
$$

where $1 / q+1 / q^{\prime}=1$. Combining (3.20) and (3.21), we immediately get

$$
\lim _{n \rightarrow \infty} \int_{B_{r}\left(x^{*}\right)}\left|f\left(x, u_{n}\right) u_{n}-f(x, u) u\right| d v_{g}=0 .
$$

A covering argument implies that for any compact set $K \subset \subset \backslash M \backslash \Sigma_{\delta}$,

$$
\lim _{n \rightarrow \infty} \int_{K}\left|f\left(x, u_{n}\right) u_{n}-f(x, u) u\right| d v_{g}=0 .
$$

Now we are proving for any compact set $K \subset \subset M \backslash \Sigma_{\delta}$,

$$
\lim _{n \rightarrow \infty} \int_{K}\left|\nabla u_{n}-\nabla u\right|^{N} d v_{g}=0 .
$$


It suffices to prove for any $x^{*} \in M \backslash \Sigma_{\delta}$, and $r: 0<r<\operatorname{dist}\left(x^{*}, \Sigma_{\delta}\right)$ given in (3.17), there holds

$$
\lim _{n \rightarrow \infty} \int_{B_{r / 2}\left(x^{*}\right)}\left|\nabla u_{n}-\nabla u\right|^{N} d x=0 .
$$

For this purpose, we take $\phi \in C_{0}^{\infty}\left(B_{r}\left(x^{*}\right)\right)$ with $0 \leq \phi \leq 1$ and $\phi \equiv 1$ on $B_{r / 2}\left(x^{*}\right)$. Obviously $\phi u_{n}$ is a bounded sequence in $E$. Inserting $\varphi=\phi u_{n}$ and $\varphi=\phi u$ into (3.16) respectively, we have

$$
\begin{aligned}
& \int_{B_{r}\left(x^{*}\right)} \phi\left(\left|\nabla u_{n}\right|^{N-2} \nabla u_{n}-|\nabla u|^{N-2} \nabla u\right)\left(\nabla u_{n}-\nabla u\right) d v_{g} \\
& \leq \int_{B_{r}\left(x^{*}\right)}\left|\nabla u_{n}\right|^{N-2} \nabla u_{n} \nabla \phi\left(u-u_{n}\right) d v_{g}+\int_{B_{r}\left(x^{*}\right)} \phi|\nabla u|^{N-2} \nabla u\left(\nabla u-\nabla u_{n}\right) d v_{g} \\
& +\int_{B_{r}\left(x^{*}\right)} \phi\left(u_{n}-u\right) f\left(x, u_{n}\right) d v_{g}+\tau_{n}\left\|\phi u_{n}\right\|_{S_{1, \tau}}+\tau_{n}\|\phi u\|_{S_{1, \tau}} .
\end{aligned}
$$

The integrals on the right side of this inequality can be estimated as below. Since $u_{n} \rightarrow u$ in $L^{p}(M)(\forall p \geq 1)$, we have by the Hölder inequality

$$
\lim _{n \rightarrow \infty} \int_{B_{r}\left(x^{*}\right)}\left|\nabla u_{n}\right|^{N-2} \nabla u_{n} \nabla \phi\left(u-u_{n}\right) d x=0 .
$$

Since $\nabla u_{n} \rightarrow \nabla u$ weakly in $\left(L^{N}(M)\right)^{N}$, there holds

$$
\lim _{n \rightarrow \infty} \int_{B_{r}\left(x^{*}\right)} \phi|\nabla u|^{N-2} \nabla u\left(\nabla u-\nabla u_{n}\right) d x=0 .
$$

From (3.21) we see $\int_{B_{r}\left(x^{*}\right)} \phi\left(u_{n}-u\right) f\left(x, u_{n}\right) d v_{g} \rightarrow 0$ as $n \rightarrow \infty$, which together with (3.25), (3.26), and $\tau_{n} \rightarrow 0$ implies that the first integral sequence of (3.24) tends to zero as $n \rightarrow \infty$. Therefore we derive (3.23) from (3.24) and an elementary inequality

$$
2^{2-N}|b-a|^{N} \leq\left\langle|b|^{N-2} b-|a|^{N-2} a, b-a\right\rangle, \quad \forall a, b \in \mathbb{R}^{N} .
$$

Since $x^{*} \in M \backslash \Sigma_{\delta}$ is arbitrary, a covering argument and (3.23) implies (3.22), which yields that $\nabla u_{n}$, up to a subsequence, converges to $\nabla u$ almost everywhere in $M$.

Let $\left(u_{n}\right)$ be a sequence such that $\nabla u_{n}(x) \rightarrow \nabla u(x)$ for almost every $x \in M$. Recall that $\left|\nabla u_{n}\right|^{N-2} \nabla u_{n}$ is bounded in $\left(L^{N-1}(M)\right)^{N}$, we can assume $\left|\nabla u_{n}\right|^{N-2} \nabla u_{n} \rightarrow V$ weakly in $\left(L^{\frac{N}{N-1}}(M)\right)^{N}$. Then $V$ must be $|\nabla u|^{N-2} \nabla u$, thanks to the almost everywhere convergence of $\nabla u_{n}$. This completes the proof of the Lemma.

\subsection{Proof of Theorems 1.3 and 1.4}

From Lemma 3.1 and Lemma 3.2, we can see that $J$ satisfies the following properties:

(i) $J \in C^{1}\left(W^{1, N}(M), \mathbb{R}\right), J(0)=0$;

(ii) There exist $\delta, \sigma>0$ such that $J(u) \geq \delta$ if $\|u\|_{S_{1, \tau}}=\sigma$.

(iii) There exists $\varphi \in W^{1, N}(M)$ such that $J(\varphi)<\delta$. 
Now we can apply the Mountain Pass Lemma [4] to obtain a positive level $c$ and a Palais-Smale sequence $\left(u_{n}\right)$ satisfying (3.15) and (3.16), where

$$
c=\inf _{\gamma \in \Gamma} \max _{u \in \gamma} J(u) \geq \delta, \quad \Gamma=\left\{\gamma \in C\left([0,1], W^{1, N}(M)\right): \gamma(0)=0, \gamma(1)=\varphi\right\} .
$$

Thanks to Lemma 3.6, $\left(u_{n}\right)$ is bounded a sequence in $W^{1, N}(M)$, and

$$
\int_{M} F\left(x, u_{n}\right) d v_{g} \leq C, \quad \int_{M} f\left(x, u_{n}\right) u_{n} d v_{g} \leq C .
$$

Up to a subsequence we can assume that

$$
\begin{aligned}
& u_{n} \rightarrow u_{0} \text { weakly in } W^{1, N}(M), u_{n} \rightarrow u_{0} \text { a. e. in } M \\
& u_{n} \rightarrow u_{0} \text { strongly in } L^{q}(M), \forall q \geq 1 .
\end{aligned}
$$

From $\left(H_{2}\right)$ and Lemma 3.6, we have

$$
F\left(x, u_{n}\right) \rightarrow F\left(x, u_{0}\right) \quad \text { in } \quad L^{1}(M)
$$

thanks to the generalized Lebesgue dominated convergence theorem, namely assume $\left(g_{n}\right),\left(h_{n}\right)$ are two measurable function sequences on $(M, g)$. Moreover $\left|g_{n}\right| \leq h_{n}$, a.e. $(n=1,2, \cdots)$; $g_{n} \rightarrow$ g, a.e.; $h_{n} \rightarrow h$, a.e.; $\int_{M} h_{n}(x) d v_{g} \rightarrow \int_{M} h(x) d v_{g}<\infty$. Then there holds

$$
\lim _{n \rightarrow \infty} \int_{M} g_{n}(x) d v_{g}=\int_{M} g(x) d v_{g} .
$$

Thus we obtain by (3.15) and (3.27)

$$
\lim _{n \rightarrow \infty} \int_{M}\left|\nabla u_{n}\right|^{N} d v_{g}=N\left(c+\int_{M} F\left(x, u_{0}\right) d v_{g}\right) .
$$

Notice that (3.16) and Lemma 3.6 lead to

$$
\int_{M}\left|\nabla u_{0}\right|^{N-2} \nabla u_{0} \nabla v d v_{g}-\int_{M} f\left(x, u_{0}\right) v d v_{g}=0, \quad \forall v \in C^{\infty}(M) .
$$

Since $C^{\infty}(M)$ is dense in $W^{1, N}(M)$, the above identity holds for all $v \in W^{1, N}(M)$. Hence $u_{0}$ is a weak solution of problem (1.7). Finally we will prove that $u_{0}$ is nontrivial. Suppose $u_{0} \equiv 0$. Then (3.28) gives

$$
\lim _{n \rightarrow \infty} \int_{M}\left|\nabla u_{n}\right|^{N} d v_{g}=N c .
$$

To proceed, we distinguish two cases:

Case 1: $f$ is subcritical.

By definition of subcritical function (1.8), $\forall \alpha: 0<\alpha<\frac{\alpha_{N}}{N c}$, there exists a constant $C$ such that

$$
\left|f\left(x, u_{n}\right)\right| \leq C+e^{\alpha\left|u_{n}\right|^{\frac{N}{N-1}}} \quad \text { for all } n .
$$


Take $q>1$ such that $q \alpha N c<\alpha_{N}$. Then

$$
\begin{aligned}
\int_{M}\left|f\left(x, u_{n}(x)\right)\right|^{q} d v_{g} & \leq C+C \int_{M} e^{q \alpha\left|u_{n}\right|^{\frac{N}{N-1}}} d v_{g} \\
& \leq C+C \int_{M} e^{q \alpha\left\|u_{n}\right\| \|_{S_{1, \tau}}^{\frac{N}{N-1}}\left|\frac{u_{n}}{\left\|u_{n}\right\|_{1, \tau}}\right|^{\frac{N}{N-1}}} d v_{g} \\
& \leq C,
\end{aligned}
$$

thanks to Theorem 1.1. Let $v=u_{n}$ in (3.16), we have by using the above estimate and $u_{n} \rightarrow 0$ in $L^{p}(M)$ for all $p \geq 1$,

$$
\begin{aligned}
\left\|u_{n}\right\|_{S_{1, \tau}}^{N} & \leq \tau_{n}\left\|u_{n}\right\|_{S_{1, \tau}}+\int_{M}\left|f\left(x, u_{n}\right) u_{n}\right| d v_{g} \\
& \leq \tau_{n}\left\|u_{n}\right\|_{S_{1, \tau}}+\left(\int_{M}\left|f\left(x, u_{n}(x)\right)\right|^{q} d v_{g}\right)^{\frac{1}{q}}\left(\int_{M}\left|u_{n}\right|^{q^{\prime}} d v_{g}\right)^{q^{\prime}} \\
& \leq \tau_{n}\left\|u_{n}\right\|_{S_{1, \tau}}+C\left\|u_{n}\right\|_{q^{\prime}} \rightarrow 0 \quad \text { as } \quad n \rightarrow \infty,
\end{aligned}
$$

where $\frac{1}{q}+\frac{1}{q^{\prime}}=1$. This contradicts (3.29). Hence $u_{0} \not \equiv 0$.

Case 2: $f$ is critical.

By definition of critical function (1.9), $\forall \epsilon>0, \exists C_{\epsilon}$ such that

$$
|f(x, s)| \leq C_{\epsilon}+e^{\left(\alpha_{0}+\epsilon\right)|s|^{\frac{N}{N-1}}} \quad \text { for all }(x, s) \in M \times \mathbb{R} .
$$

By Lemma 3.3, $c<\frac{1}{N}\left(\frac{\alpha_{N}}{\alpha_{0}}\right)$. Clearly $\left\|u_{n}\right\|_{S_{1, \tau}} \rightarrow N c$ thanks to (3.29) and $u_{n} \rightarrow 0$ in $L^{p}(M)$ for all $p \geq 1$. We choose $\epsilon>0$ sufficiently small and $q>1$ sufficiently close to 1 such that $q\left(\alpha_{0}+\epsilon\right)\left\|u_{n}\right\|_{S_{1, \tau}}^{\frac{N}{N-1}}<\alpha_{N}$ for sufficiently large $n$. Then

$$
\begin{aligned}
\int_{M}\left|f\left(x, u_{n}(x)\right)\right|^{q} d v_{g} & \leq 2^{q} C_{\epsilon}^{q}+2^{q} \int_{M} e^{q\left(\alpha_{0}+\epsilon\right)\left|u_{n}\right|^{\frac{N}{N-1}}} d v_{g} \\
& \leq 2^{q} C_{\epsilon}^{q}+2^{q} \int_{M} e^{q \alpha\left\|u_{n}\right\|_{S_{1, \tau}}^{\frac{N}{N-1}}\left|\frac{u_{n}}{\left\|u_{n}\right\|_{1, \tau}}\right|^{\frac{N}{N-1}}} d v_{g} \\
& \leq C .
\end{aligned}
$$

As in Case 1, we obtain $\left\|u_{n}\right\|_{S_{1, \tau}} \rightarrow 0$ which contradicts (3.29). Hence $u_{0} \not \equiv 0$. This completes the proof of Theorems 1.3 and 1.4.

\subsection{An example of maximizer}

In this subsection, we will give an example of maximizer. In view of Theorem 1.1, one has for all $\alpha \leq \alpha_{N, m}$

$$
\Lambda_{\alpha}=\sup _{\|u\|_{S_{m, \tau}} \leq 1} \int_{M} e^{\alpha|u|^{\frac{N}{N-m}}} d v_{g}<\infty .
$$

Furthermore we have the following: 
Proposition 3.7 Assume $0<m<N$. For any $\alpha$ : $0<\alpha<\alpha_{N, m}$, there exists a function $u_{\alpha} \in W^{m, N / m}(M)$ with $\|u\|_{S_{m, \tau}} \leq 1$ such that

$$
\int_{M} e^{\alpha\left|u_{\alpha}\right| \frac{N}{N-m}} d v_{g}=\sup _{\|u\|_{S_{m, \tau}} \leq 1} \int_{M} e^{\alpha|u|^{\frac{N}{N-m}}} d v_{g} .
$$

Moreover $u_{\alpha}$ is a weak solution of the equation

$$
\left\{\begin{array}{l}
-\Delta^{k-1}\left(\operatorname{div}\left(\left|\nabla \Delta^{k-1} u_{\alpha}\right|^{\frac{N}{m}-2} \nabla \Delta^{k-1} u_{\alpha}\right)\right)+\tau\left|u_{\alpha}\right|^{\frac{N}{m}-2} u_{\alpha}= \\
\frac{1}{\lambda_{\alpha}}\left|u_{\alpha}\right|^{\frac{N}{N-m}-2} u_{\alpha} e^{\alpha\left|u_{\alpha}\right|^{\frac{N}{N-m}}} \text { when } m=2 k-1, k=1,2, \cdots, \\
\Delta^{k}\left(\left|\Delta^{k} u_{\alpha}\right|^{\frac{N}{m}-2} \Delta^{k} u_{\alpha}\right)+\tau\left|u_{\alpha}\right|^{\frac{N}{m}-2} u_{\alpha}= \\
\frac{1}{\lambda_{\alpha}}\left|u_{\alpha}\right|^{\frac{N}{N-m}-2} u_{\alpha} e^{\alpha\left|u_{\alpha}\right|^{\frac{N}{N-m}}} \text { when } m=2 k, k=1,2, \cdots, \\
\lambda_{\alpha}=\int_{M}\left|u_{\alpha}\right|^{\frac{N}{N-m}} e^{\alpha\left|u_{\alpha}\right|^{\frac{N}{N-m}}} d v_{g}, \quad\left\|u_{\alpha}\right\|_{S_{m, \tau}}=1 .
\end{array}\right.
$$

In particular, when $m=1, u_{\alpha}$ can be further chosen nonnegative and thus satisfies

$$
-\Delta_{N} u_{\alpha}+\tau u_{\alpha}^{N-1}=\frac{1}{\lambda_{\alpha}} u_{\alpha}^{\frac{1}{N-1}} e^{\alpha u_{\alpha}^{N-1}} \quad \text { in } M .
$$

Remark 3.8 In the case when $m=1$, it is easy to see that $0<\lambda_{\alpha}<\alpha_{N}$ for any $0<\alpha<\alpha_{N}$. Proposition 3.8 particularly gives a positive solution of the $N$-Laplacian equation

$$
-\Delta_{N} u+\tau|u|^{N-2} u=f(x, u(x)) \text { in } M,
$$

where $f(x, u)=\frac{1}{\lambda_{\alpha}}|u|^{\frac{1}{N-1}-1} u e^{\alpha|u|^{N-1}}$ is critical, $\lambda_{\alpha}$ is defined by (3.30). We calculate for all $s>0$,

$$
F(x, s)=\int_{0}^{s} f(x, t) d t=\frac{N-1}{\alpha \lambda_{\alpha} N}\left(e^{\alpha s^{\frac{N}{N-1}}}-1\right) .
$$

It can be easily checked that $f$ satisfies $\left(H_{1}\right),\left(H_{2}\right)$, and $\left(H_{3}\right)$. But when $N \geq 3$,

$$
\frac{N F(x, s)}{s^{N}} \rightarrow+\infty \quad \text { as } \quad s \rightarrow 0+,
$$

thus $\left(H_{4}\right)$ does not hold. This possibly yields a new method of studying positive solutions of the above $N$-Laplacian equation with $f(x, u)$ behaves like $e^{\alpha|u|^{N-1}}$ as $|u| \rightarrow \infty$.

Remark 3.9 For compactness analysis of the above equations, particularly for extremal functions of the Trudinger-Moser inequality on manifolds, we refer the reader to [11, 12].

Proof of Proposition 3.7: It is easy to see that

$$
\sup _{\|u\|_{S_{m, \tau}}=1} \int_{M} e^{\alpha|u|^{\frac{N}{N-m}}} d v_{g}=\sup _{\|u\|_{S_{m, \tau}} \leq 1} \int_{M} e^{\alpha|u|^{N-m}} d v_{g}=\Lambda_{\alpha} .
$$

Take a function sequence $u_{k}$ with $\left\|u_{k}\right\|_{S_{m, \tau}}=1$ such that

$$
\int_{M} e^{\alpha\left|u_{k}\right|^{\frac{N}{N-m}}} d v_{g} \rightarrow \Lambda_{\alpha} \quad \text { as } \quad k \rightarrow \infty .
$$


Up to a subsequence, we can assume

$$
\begin{array}{lll}
u_{k} \rightarrow u_{\alpha} & \text { weakly in } & W^{m, \frac{N}{m}}(M) \\
u_{k} \rightarrow u_{\alpha} & \text { strongly in } \quad L^{p}(M), \forall p \geq 1 \\
u_{k} \rightarrow u_{\alpha} & \text { a.e. in } \quad M .
\end{array}
$$

It follows that

$$
\begin{aligned}
\int_{M}\left|\nabla^{m} u_{\alpha}\right|^{\frac{N}{m}} d v_{g} & =\lim _{k \rightarrow \infty} \int_{M}\left|\nabla^{m} u_{\alpha}\right|^{\frac{N}{m}-2} \nabla^{m} u_{\alpha} \nabla^{m} u_{k} d v_{g} \\
& \leq \limsup _{k \rightarrow \infty}\left(\int_{M}\left|\nabla^{m} u_{\alpha}\right|^{\frac{N}{m}} d v_{g}\right)^{\frac{N-m}{N}}\left(\int_{M}\left|\nabla^{m} u_{k}\right|^{\frac{N}{m}} d v_{g}\right)^{\frac{m}{N}} \\
& \leq\left(\int_{M}\left|\nabla^{m} u_{\alpha}\right|^{\frac{N}{m}} d v_{g}\right)^{\frac{N-m}{N}} .
\end{aligned}
$$

Hence we obtain $\left\|u_{\alpha}\right\|_{S_{m, \tau}} \leq 1$, thanks to $u_{k} \rightarrow u_{\alpha}$ strongly in $L^{p}(M)$ for all $p \geq 1$. On the other hand, the mean value theorem implies that

$$
e^{\alpha\left|u_{k}\right| \frac{N}{N-m}}-e^{\alpha\left|u_{\alpha}\right|^{\frac{N}{N-m}}}=e^{\xi} \alpha\left(\left|u_{k}\right|^{\frac{N}{N-m}}-\left|u_{\alpha}\right|^{\frac{N}{N-m}}\right)
$$

for some $\xi(x)$ lies between $\left|u_{k}(x)\right|$ and $\left|u_{\alpha}(x)\right|$, and that

$$
\left|u_{k}\right|^{\frac{N}{N-m}}-\left|u_{\alpha}\right|^{\frac{N}{N-m}}=\frac{N}{N-m} \zeta^{\frac{m}{N-m}}\left(\left|u_{k}\right|-\left|u_{\alpha}\right|\right)
$$

for some $\zeta(x)$ lies between $\left|u_{k}(x)\right|$ and $\left|u_{\alpha}(x)\right|$. Notice that $u_{k}$ is bounded in $L^{q}(M), u_{k} \rightarrow u_{\alpha}$ in $L^{q}(M)$ for all $q \geq 1$, and $e^{\alpha\left|u_{k}\right|^{N-1}}$ is bounded in $L^{r}(M)$ for some $r>1$, applying the Hölder inequality to the above two equalities, one can derive that

$$
\int_{M} e^{\alpha\left|u_{\alpha}\right|^{N-m}} d v_{g}=\lim _{k \rightarrow \infty} \int_{M} e^{\alpha\left|u_{k}\right|^{\frac{N}{N-m}}} d v_{g}=\Lambda_{\alpha} .
$$

Hence we obtain by (3.31)

$$
\int_{M} e^{\alpha\left|u_{\alpha}\right|^{\frac{N}{N-m}}} d v_{g}=\sup _{\|u\|_{S_{m, \tau}} \leq 1} \int_{M} e^{\alpha|u|^{\frac{N}{N-m}}} d v_{g}
$$

and $\left\|u_{\alpha}\right\|_{S_{m, \tau}}=1$. Clearly $u_{\alpha}$ is a critical point of the functional $J_{\alpha}(u)=\int_{M} e^{\alpha|u|^{\frac{N}{N-m}}} d v_{g}$ under the constraint $\|u\|_{S_{m, \tau}}=1$. A straightforward calculation shows $u_{\alpha}$ satisfies the Euler-Lagrange equation (3.30).

When $m=1$, notice that $u \in W^{1, N}(M)$ implies $|u| \in W^{1, N}(M)$ and \|\|$u\|\|_{S_{1, \tau}} \leq\|u\|_{S_{1, \tau}}$. If $u_{\alpha}$ satisfies (3.32) and $\left\|u_{\alpha}\right\|_{S_{1, \tau}}=1$, then so does $\left|u_{\alpha}\right|$. Hence $u_{\alpha}$ can be chosen such that $u_{\alpha} \geq 0$.

Acknowledgement The authors thank the referee for improvement of this paper, especially the proof of the modified Adams-Fontana inequality. The second named author is partly supported by the program for NCET. 


\section{References}

[1] D. Adams: A sharp inequality of J. Moser for higher order derivatives. Ann. Math., 128: 385-398, 1988.

[2] Adimurthi: Existence of positive solutions of the semilinear Dirichlet problem with critical growth for the $n$ Laplacian. Ann. Scuola Norm. Sup. Pisa Cl. Sci., 17: 393-413, 1990.

[3] Adimurthi and Y. Yang: An interpolation of Hardy inequality and Trudinger-Moser inequality in $\mathbb{R}^{N}$ and its applications. International Mathematics Research Notices, 2010. doi:10.1093/imrn/rnp194

[4] A. Ambrosetti and P. H. Rabinowitz: Dual variational methods in critical point theory and applications. J. Functional Anal., 14: 349-381, 1973.

[5] P. Cherrier: Problemes de Neumann non linearires sur les varietes riemanniennes. C. R. Acad. Sc. Paris, Serie A, 292: 637-640, 1981.

[6] P. Cherrier: Melleures constants dans des inegalites relatives aux espaces de Sobolev. Bull. Sci. Math 2, 108 225-262, 1984

[7] D. G de Figueiredo, J. M. do Ó and B. Ruf: On an inequality by Trudinger and J. Moser and related elliptic equations. Comm. Pure Appl. Math., 55: 135-152, 2002.

[8] D. G. de Figueiredo, O. H. Miyagaki and B. Ruf: Elliptic equations in $\mathbb{R}^{2}$ with nonlinearities in the critical growth range. Calc. Var., 3: 139-153, 1995.

[9] J. M. do Ó: Semilinear Dirichlet problems for the $N$-Laplacian in $\mathbb{R}^{N}$ with nonlinearities in the critical growth range. Differential and Integral Equations, 9: 967-979, 1996

[10] L. Fontana: Sharp borderline Sobolev inequalities on compact Riemannian manifolds. Comm. Math. Helv., 68: 415- 454, 1993.

[11] Y. Li: Extremal functions for the Moser-Trudinger inequalities on compact Riemannian manifolds. Science in China, Ser. A, 48: 618-648, 2005.

[12] Y. Li and C. Ndiaye: Extremal functions for Moser-Trudinger type inequality on compact closed 4-manifolds. J. Geom. Anal. 17: 669-699, 2007.

[13] J. Moser: A sharp form of an Inequality by N.Trudinger. Ind. Univ. Math. J., 20: 1077-1091, 1971.

[14] Z. Nehari: On a class of non-linear second order differential equations. Trans. AMS, 95: 101-123, 1960

[15] S. Pohozaev: The Sobolev embedding in the special case $p l=n$. Proceedings of the technical scientific conference on advances of scientific reseach 1964-1965, Mathematics sections, 158-170, Moscov. Energet. Inst., Moscow, 1965.

[16] N. S. Trudinger: On embeddings into Orlicz spaces and some applications. J. Math. Mech., 17: 473-484, 1967.

[17] Y. Yang: Extremal functions for Moser-Trudinger inequalities on 2-dimensional compact Riemannian manifolds with boundary. Internat. J. Math., 17: 313-330, 2006.

[18] Y. Yang and L. Zhao: A class of Adams-Fontana type inequalities and related functionals on manifolds. Nonlinear Diff. Eqn. Appl., 17: 119-135, 2010. 